\title{
LOCAL BEHAVIOR OF SOLUTIONS OF QUASILINEAR ELLIPTIC EQUATIONS WITH GENERAL STRUCTURE
}

\author{
J. M. RAKOTOSON AND WILLIAM P. ZIEMER
}

\begin{abstract}
This paper is motivated by the observation that solutions to certain variational inequalities involving partial differential operators of the form $\operatorname{div} A(x, u, \nabla u)+B(x, u, \nabla u)$, where $A$ and $B$ are Borel measurable, are solutions to the equation $\operatorname{div} A(x, u, \nabla u)+B(x, u, \nabla u)=\mu$ for some nonnegative Radon measure $\mu$. Among other things, it is shown that if $u$ is a Hölder continuous solution to this equation, then the measure $\mu$ satisfies the growth property $\mu[B(x, r)] \leq M r^{n-p+\varepsilon}$ for all balls $B(x, r)$ in $\mathbf{R}^{n}$. Here $\varepsilon$ depends on the Hölder exponent of $u$ while $p>1$ is given by the structure of the differential operator. Conversely, if $\mu$ is assumed to satisfy this growth condition, then it is shown that $u$ satisfies a Harnack-type inequality, thus proving that $u$ is locally bounded. Under the additional assumption that $A$ is strongly monotonic, it is shown that $u$ is Hölder continuous.
\end{abstract}

\section{INTRODUCTION}

In this paper we investigate the behavior of weak solutions of quasilinear equations of second order in an open set $\Omega \subset \mathbf{R}^{N}$. The equations are of the form

$$
-\operatorname{div} A(x, u, \nabla u)+B(x, u, \nabla u)=T,
$$

where $T$ is a distribution that will be specified below. The case in which $T$ is a measure will be of special interest to us because this situation arises in the study of variational inequalities. Indeed, let $\psi$ be an obstacle defined on $\Omega$, and consider

$$
I(v)=\int_{\Omega} F(x, v, \nabla v) d x,
$$

where $F=F(x, \eta, \xi)$ is an integrand with suitable properties. Let

$$
\sigma=\inf _{v \in K} I(v),
$$

where $K$ is the subset of the Sobolev space $W^{1, p}(\Omega)$ consisting of all $v$ that agree with a prescribed boundary function $\theta$ on $\partial \Omega$ and $v(x) \geq \psi(x)$ for

Received by the editors September 5, 1988 .

1980 Mathematics Subject Classification (1985 Revision). Primary 35J20; Secondary 35D10.

Research supported in part by a grant from the National Science Foundation. 
almost all $x \in \Omega$. If $u$ is the extremal of problem (1.3), then it is well known that $u$ satisfies an inequality of the form

$$
\int_{\Omega} \frac{\partial F}{\partial \xi}(x, u, \nabla u) \cdot \nabla \varphi+\frac{\partial F}{\partial \eta}(x, u, \nabla u) \varphi d x \geq 0
$$

for all $\varphi \in W_{0}^{1, p}(\Omega)$ with $\varphi(x) \geq \psi(x)-u(x)$ for a.e. $x \in \Omega$. This is a special case of the weak inequality

$$
\int_{\Omega} A(x, u, \nabla u) \cdot \nabla \varphi+B(x, u, \nabla u) \varphi d x \geq 0 .
$$

In particular, (1.4) and (1.5) hold for all nonnegative $\varphi \in W_{0}^{1, p}(\Omega)$ and thus define a nonnegative distribution. Consequently, $u$ is a solution of the equation

$$
-\operatorname{div} A(x, u, \nabla u)+B(x, u, \nabla u)=\mu,
$$

where $\mu$ is some nonnegative measure. It was shown in [MZ] that if $A$ and $B$ satisfy the structure (1.7), (1.8), (1.9) below, then the solution of the obstacle problem is continuous at a point $x_{0}$ provided that the obstacle satisfies a weak regularity condition as $x_{0}$, a so-called Wiener condition reminiscent of the condition considered in [GZ]. In particular, the results of [MZ] imply that if $\psi$ is Hölder continuous, then the solution $u$ is also Hölder continuous. Variational inequalities were also investigated in [RT], and there Hölder regularity was established by different techniques. In order to gain a better understanding of such variational inequalities, including questions of differentiability, we begin an investigation in this paper of equation (1.6). Investigations in problems of this type were initiated in [LS], where the Laplace operator was considered.

We assume the functions $A$ and $B$ are, respectively, vector- and scalarvalued Borel functions defined on $\Omega \times(m, M) \times \mathbf{R}^{N},-\infty<m \leq M<+\infty$, that satisfy the following structure.

For a.e. $x \in \Omega, \forall \eta \in(m, M), \forall \xi \in \mathbf{R}^{N}$, we have

$$
A(x, \eta, \xi) \cdot \xi \geq \nu_{0}(x)|\xi|^{p}-\nu_{1}(x),
$$

where $1<p<N, \nu_{0}$ is a positive continuous function on $\Omega$, and $\nu_{1} \in$ $L_{\text {loc }}^{N / p+\varepsilon}(\Omega)$, with $\varepsilon_{1}>0$;

$$
|A(x, \eta, \xi)| \leq c_{0}(x)|\xi|^{p-1}+a_{0}(x)
$$

for $c_{0} \in L_{\mathrm{loc}}^{\infty}(\Omega), c_{0} \geq 0$, and $a_{0} \in L^{N /(p-1)+\varepsilon_{2}}(\Omega), \varepsilon_{2}>0$;

$$
|B(x, \eta, \xi)| \leq c_{1}(x)|\xi|^{p}+f_{0}(x),
$$

where $c_{1} \in L_{\text {loc }}^{\infty}(\Omega), c_{1} \geq 0$, and $f_{0} \in L_{\text {loc }}^{N / p+\varepsilon_{3}}(\Omega), \varepsilon_{3}>0$. In the sequel, we will also consider the structure condition

$$
|B(x, \eta, \xi)| \leq c_{1}(x)|\xi|^{p-1}+f_{0}(x),
$$

where $c_{1} \in L_{\mathrm{loc}}^{\infty}(\Omega), c_{1} \geq 0$, and $f_{0} \in L_{\mathrm{loc}}^{N / p+\varepsilon_{3}}(\Omega), \varepsilon_{3}>0$. 
The results of the paper are summarized as follows. In $\S 2$ we first treat equation (1.1) with $T \in W^{-1, q_{0}}(\Omega), q_{0}>N /(p-1)$, and prove weak Harnack inequalities for subsolutions and supersolutions that are analogous to those established in [T] when $T=0$. In $\S 3$ we focus on equation (1.1) where $T$ is now assumed to be a nonnegative measure $\mu$. We first show (Theorem 3.2) that if a solution of this equation is locally Hölder continuous, then the measure $\mu$ must satisfy the growth property $\mu[B(x, r)] \leq M r^{N-p+\varepsilon}$ for some $\varepsilon>0$ and all balls $B(x, r)$. We then pursue the converse of this result. Under the assumption that $\mu$ satisfies $\mu[B(x, r)] \leq M r^{N-p+\varepsilon}$ for all balls, we show that solutions $u \in W_{\text {loc }}^{1, p}(\Omega)$ of (1.1) with structure (1.7), (1.8), (1.10) are locally bounded (Theorem 2.10) and that $u \in W_{\text {loc }}^{1, q}(\Omega)$ for some $q>p$ (Corollary 2.13). It is also shown that $u$ satisfies a Harnack-type inequality (Theorem 2.14) which bears some resemblance to the classical one for solutions when $\mu=0$. Under the additional assumption that $A$ is strongly monotonic (see Definition 3.6) Theorem 3.7 establishes that weak solutions are locally Hölder continuous.

Before beginning with the analysis, we give some examples of (1.1) that have been treated in other contexts.

1.1. Example. The following example appears in Aerodynamics and has been recently treated in [MRS1, MRS2].

$$
-\frac{\partial^{2} u}{\partial x_{2}^{2}}-\frac{1-\bar{M}^{2}(u)}{\rho^{2}(u)} \frac{\partial^{2} u}{\partial x_{1}^{2}}+\frac{1}{u}\left[\left(\frac{\partial u}{\partial x_{2}}\right)^{2}+\frac{1+\gamma \bar{M}^{4}(u)}{\rho^{2}(u)}\left(\frac{\partial u}{\partial x_{i}}\right)^{2}\right]=0
$$

$\Omega \subset \mathbf{R}^{2}$.

The function $\bar{M}$ (called the mach number) is defined by

$$
\bar{M}(u)=u \sqrt{\frac{2}{\gamma+1} \cdot \frac{1}{1-(\gamma-1) /(\gamma+1)}} u^{2}
$$

and

$$
\rho(u)=\left(1-\frac{\gamma-1}{\gamma+1} u^{2}\right)^{1 /(\gamma-1)},
$$

where $u$ is the velocity of a fluid crossing $\Omega, \rho$ is the density fo this fluid (see [MRS1, MRS2] for details). Problem (1.11) can be written in the form of (1.1). The velocity at the boundary is given: $u=g$ on $\partial \Omega$ and satisfies $0<\theta \leq g<1$. It is proved in [MRS1] that there exists at least a solution $u$ in $H^{1}(\Omega)$ satisfying $\theta \leq u(x)<1$ a.e. Here, $m=\theta, M=1$.

\subsection{Example.}

$$
\left\{\begin{array}{l}
-\operatorname{div}\left(\nu(u)|\nabla u|^{p-2} \nabla u\right)+\left(e^{u}-1\right)|\nabla u|^{p}+u f^{2}(x)=T(x) \text { in } \Omega, \\
u=0 \text { on } \partial \Omega .
\end{array}\right.
$$

Here,

$$
\nu(u)=\frac{1}{1+|u|^{p}}, \quad T \in L^{N / p+\varepsilon}(\Omega), \quad f \in L^{\infty}(\Omega) .
$$


It is proved in $[R]$ using the techniques of relative rearrangement that such equation has a bounded solution, and therefore we can take

$$
\begin{gathered}
m=-\operatorname{ess}_{\Omega} \sup |u|, \quad M=\operatorname{ess}_{\Omega} \sup |u|, \\
\nu_{0}=\operatorname{Min}_{|\eta| \leq M} \nu(\eta), \quad c_{1}=\operatorname{Max}_{(\eta) \leq M}\left|e^{\eta}-1\right|,
\end{gathered}
$$

and $f_{0}(x)=M f^{2}(x)$. All problems considered in [R, RT, MRS1, MRS2, L] are covered by these assumptions.

1.3. Example. In this example, we consider an unbounded domain: $\Omega=\mathbf{R}^{N}$.

$$
\left\{\begin{array}{l}
-\operatorname{div}\left(\frac{\nu(u)}{1+|x|}|\nabla u|^{p-2} \nabla u\right)+\frac{e^{u}|\nabla u|^{p}}{1+|x|^{\alpha}}=T(x), \\
u \in W^{1, p}\left(\mathbf{R}^{N}\right), \quad T \in L^{\infty}, \quad \nu(u)=\frac{1}{1+|u|}, \quad \alpha \geq 0 .
\end{array}\right.
$$

\section{Preliminaries}

Throughout, we will adopt the convention of using $C$ as a constant that may change from line to line in a proof. For $p \geq 1$, we denote by $p^{\prime}$ the conjugate of $p$. The integral average is denoted by

$$
f_{E} u d x=|E|^{-1} \int_{E} u(x) d x .
$$

In this section we fix a bounded open set $\Omega_{1}$ relatively compact in $\Omega$. Choose numbers $q$ and $q_{1}$ such that

$$
\frac{N}{p-1}<q<q_{1}\left(\frac{2 N p^{\prime}}{p q_{1}+N p^{\prime}}\right)<q_{1} .
$$

In this section we also consider a distribution $T$ in (1.1) such that

$$
T \in W^{-1, q_{1}}\left(\Omega_{1}\right) \text {. }
$$

By the assumptions on $\nu_{1}, a_{0}, f_{0}$ we have

$$
T, f_{0} \in W^{-1, q_{1}}\left(\Omega_{1}\right), \quad a_{0} \in L^{q_{1}}\left(\Omega_{1}\right), \nu_{1} \in L^{q_{1} / p^{\prime}}\left(\Omega_{1}\right) .
$$

Let $\varepsilon_{0}= \pm$. Since $\varepsilon_{0} T+f_{0} \in W^{-1, q}\left(\Omega_{1}\right)$, there exists $f \in L^{q}\left(\Omega_{1} ; \mathbf{R}^{N}\right)$ such that $\varepsilon_{0} T+f_{0}=\operatorname{div} f$. We set

$$
\nu_{0}=\min _{\bar{\Omega}_{1}} \nu_{0}(x), \quad c_{0}=\operatorname{ess}_{\Omega_{1}} \sup c_{0}(x), \quad c_{1}=\operatorname{ess}_{\Omega_{1}} \sup c_{1}(x)+1 .
$$

We consider $x_{0} \in \Omega_{1}$, and for all $r>0$ such that $B\left(x_{0}, 4 r\right)$ is contained in $\bar{\Omega}_{1}$, we define

$$
\rho(r)=r^{1-N /(p-1) q} I\left(\Omega_{1}\right)
$$

and

$$
I\left(\Omega_{1}\right)=\|T\|_{W^{-1 . q}\left(\Omega_{1}\right)}+\left\|f_{0}\right\|_{L^{N / p}\left(\Omega_{1}\right)}+\left\|a_{0}\right\|_{L^{N / p-1}\left(\Omega_{1}\right)}+\left\|\nu_{1}\right\|_{L^{N / p-1)}\left(\Omega_{1}\right)} .
$$


Define also the function

$$
b_{r}(x)=\frac{1}{\rho(r)^{p}}\left[\left(f(x)+a_{0}(x)\right)^{p^{\prime}}+\nu_{1}(x)\right] \quad \text { if } I\left(\Omega_{1}\right) \neq 0
$$

and

$$
b_{r}(x)=0 \text { if } I\left(\Omega_{1}\right)=0 .
$$

By an application of Hölder's inequality, observe that there exists a constant $c_{3}$ such that for all $x_{0} \in \Omega$, and all $r>0$ with $B\left(x_{0}, 4 r\right) \subset \bar{\Omega}_{1}$ we have

$$
\left\|b_{r}\right\|_{L^{q / p^{\prime}}\left(B\left(x_{0}, 4 r\right)\right)} \leq c_{3}^{\prime} \text {. }
$$

We now proceed to obtain weak Harnack inequalities for (1.1) with $T$ as in (2.2) analogous to those obtained in [T] where $T=0$. Our techniques are similar to those in [T], and therefore we will provide only those parts of the proofs that will be necessary for the development of the remainder of this paper.

2.1. Theorem. Let $u \in W_{\text {loc }}^{1, p}(\Omega)$ be a nonnegtive, locally bounded subsolution of (1.1) with structure (1.7), (1.8), (1.9) and $T \in W_{\mathrm{loc}}^{-1, q_{0}}(\Omega), q_{0}>N /(p-1)$. Then, for all $x_{0} \in \Omega$ and $r>0$ such that $B\left(x_{0}, 4 r\right) \subset \bar{\Omega}_{1} \subset \Omega$, and for all $p_{0}>p-1$, we have

$$
\sup _{B\left(x_{0}, r\right)} u \leq C\left(r^{-N / p_{0}}\|u\|_{L^{p_{0}\left(B\left(x_{0}, 2 r\right)\right)}}+\rho(r)\right) .
$$

The constant $C$ depends only on the structure, the bound on $u$, and the fixed set $\Omega_{1}$.

2.2. Theorem. Let $u \in W_{\mathrm{loc}}^{1, p}(\Omega)$ be a nonnegative, locally bounded supersolution of (1.1) with structure (1.7), (1.8), (1.9). Then, for all $x_{0} \in \Omega$ and $r>0$ such that $B\left(x_{0}, 4 r\right) \subset \bar{\Omega}_{1} \subset \Omega$, and all $p_{0}$ such that $0<p_{0}<\widehat{N}(p-1) /(\widehat{N}-p)$ (here $\widehat{N}=N$ if $p<N, N<\widehat{N}<q(p-1)$ if $p=N)$, we have

$$
r^{-N / p_{0}}\|u\|_{L^{p_{0}\left(B\left(x_{0} 2 r\right)\right)}} \leq C\left(\min _{B\left(x_{0}, r\right)} u+\rho(r)\right) .
$$

The constant $C$ depends only on the structure, the bound on $u$, and the fixed set $\Omega_{1}$.

The proofs of these theorems require the following familiar test functions. Let $\mu_{1}>0, \mu_{2}>0$ be arbitrary. For $\beta \in \mathbf{R}$, and $t>0$, define

$$
\sigma(t)=\frac{1}{\mu_{1}} \exp \left(\frac{\mu_{2}}{\mu_{1}}(\operatorname{sign} \beta) t\right) t^{\beta} .
$$

Then $\sigma$ is a solution of

$$
(\operatorname{sign} \beta) \mu_{1} \sigma^{\prime}-\mu_{2} \sigma=|\beta| t^{\beta-1} \exp \left(\frac{\mu_{2}}{\mu_{1}}(\operatorname{sign} \beta) t\right) .
$$


So for all number $b>0$, we can find two numbers $c_{b}>0, c_{b}^{\prime}>0$ depending only on $b, \mu_{1}$, and $\mu_{2}$ such that

$$
\begin{array}{r}
(\operatorname{sign} \beta) \mu_{1} \sigma^{\prime}-\mu_{2} \sigma \geq c_{b}|\beta| t^{\beta-1} \\
0 \leq \sigma(t) \leq c_{b}^{\prime} t^{\beta}
\end{array} \text { for all } t \in(0, b] .
$$

For $x_{0} \in \Omega_{1}$ let $\eta \in C_{0}^{\infty}(\Omega)$ be such that $0 \leq \eta \leq 1$ and $\operatorname{spt} \eta \subset B\left(x_{0}, 4 r\right) \subset$ $\bar{\Omega}_{1}$. Let $0<\lambda<1$ (where $\lambda$ tends to zero at the end of the proof). Define

$$
\begin{gathered}
\bar{u}=u+\rho(r)+\lambda, \quad \gamma=\beta+p-1, \quad \beta \neq 0, \\
w(x)= \begin{cases}\bar{u}^{\gamma / p} & \text { if } \gamma \neq 0, \\
\log \bar{u} & \text { if } \gamma=0 .\end{cases}
\end{gathered}
$$

The following energy inequality is fundamental to the development.

2.3. Lemma. There exists a constant $C>0$ such that

$$
\int_{\Omega}|\eta \nabla w|^{p} d x \leq \begin{cases}|\gamma|^{p} C h_{0}(|\beta|) \int_{\Omega}\left(b_{r} \eta^{p}+|\nabla \eta|^{p}\right) w^{p} d x & \text { if } \gamma \neq 0 \\ C h_{0}(|\beta|) \int_{\Omega}\left(b_{r} \eta^{p}+|\nabla \eta|^{p}\right) d x & \text { if } \gamma=0\end{cases}
$$

where $h_{0}(|\beta|)=1+1 /|\beta|+1 /|\beta|^{p}+1 /|\beta|^{p^{\prime}}$.

Proof. Define $\varphi=\eta^{p}(\operatorname{sign} \beta) \sigma(\bar{u})$, where $\sigma$ is the function defined in relation (2.5) with

$$
\mu_{1}=\min _{\bar{\Omega}_{1}} \nu_{0}(x), \quad \mu_{2}=\operatorname{ess}_{\Omega_{1}} \sup c_{1}(x)+1
$$

Since $u$ is locally bounded, we can find $C>0$ such that

$$
\begin{gathered}
0<\sigma(\bar{u}) \leq C \bar{u}^{\beta}, \quad\left|\sigma^{\prime}(\bar{u})\right| \leq C|\beta| \bar{u}^{\beta-1}+C \bar{u}^{\beta}, \\
(\operatorname{sign} \beta) \mu_{0} \sigma^{\prime}(\bar{u})-C \sigma(\bar{u}) \geq C|\beta| \bar{u}^{\beta-1} .
\end{gathered}
$$

With this choice of $\varphi$ employed in (1.1) with its structure, we obtain

$$
\begin{aligned}
& \int|\nabla u|^{p} \eta^{p}\left[(\operatorname{sign} \beta) \mu_{1} \sigma^{\prime}(\bar{u})-\mu_{2} \sigma(\bar{u})\right] d x \\
& \leq p C_{0} \int|\nabla u| \eta^{p-1}|\nabla u|^{p-1} \sigma(\bar{u}) d x+p \int|\nabla \eta| \eta^{p-1} a_{0}(x) \sigma(\bar{u}) d x \\
& \quad+\int \eta^{p} \nu_{1}\left|\sigma^{\prime}(\bar{u})\right| d x+\int \eta^{p} f_{0} \sigma(\bar{u}) d x+\langle T, \varphi\rangle
\end{aligned}
$$

The last two terms of (2.9) can be written as

$$
\int \eta^{p} \sigma(\bar{u}) f_{0} d x+\langle T, \varphi\rangle=\left\langle f_{0}+(\operatorname{sign} \beta) T, \eta^{p} \sigma(\bar{u})\right\rangle .
$$

Using relation (2.3) we can find $f \in L^{q}\left(\Omega_{1}\right)$ such that if $f_{0}+(\operatorname{sign} \beta) T=\operatorname{div} f$, 
where $f$ does not depend on $\beta$. Using (2.8) we obtain

$$
\begin{aligned}
& |\beta| \int|\nabla u|^{p} \eta^{p} \bar{u}^{\beta-1} d x \leq C_{0} \int|\nabla u|^{p-1} \eta^{p-1}|\nabla \eta| \bar{u}^{\beta} d x \\
& \quad+c_{11} \int \eta^{p-1}|\nabla \eta| a_{0}(x) \bar{u}^{\beta} d x \\
& \quad+c_{12}(1+|\beta|) \int \nu_{1} \eta^{p} \bar{u}^{\beta-1} d x+c_{13} \int \eta^{p-1}|\nabla \eta| f \bar{u}^{\beta} d x \\
& \quad+c_{14}|\beta| \int f|\nabla u| \eta^{p} \bar{u}^{\beta-1} d x+c_{15} \int f|\nabla u| \eta^{p} \bar{u}^{\beta-1} d x
\end{aligned}
$$

Each term of the right side can be analyzed in a manner similar to [T] to obtain the conclusion of the lemma.

With the basic energy inequality of Lemma 2.3 , it is possible to obtain Theorems 2.1 and 2.2 as in [T], whose argument depends critically on the Sobolev inequality and the John-Nirenberg inequality [JM].

Theorems 2.1 and 2.2 yield the Harnack inequality

$$
\sup _{B\left(x_{0}, r\right)} u \leq C\left[\inf _{B\left(x_{0}, r\right)} u+\rho(r)\right]
$$

whenever $B\left(x_{0}, r\right) \subset \Omega_{1} \subset \Omega$. As $\Omega_{1}$ is arbitrary, we obtain as a consequence

2.4. Theorem. Let $u \in W_{\mathrm{loc}}^{1, p}(\Omega)$ be a nonnegative, locally bounded solution of (1.1) with structure $(1.7),(1.8),(1.9)$. Then $u$ is locally Hölder continuous in $\Omega$.

2.5. Remark. If $u \in W_{\mathrm{loc}}^{1, p}(\Omega)$ is a solution of (1.1) with structure (1.7), (1.8), and (1.10), then the methods above can be modified so that Theorems 2.1 and 2.2 hold without the assumption that $u$ is bounded. With this structure, subsolutions and supersolutions are bounded above and below, respectively.

\section{The equation With $T$ as a measure}

In this section we consider equation (1.1) with $T$ assumed to be a nonnegative Radon measure $\mu$ and seek conditions on $\mu$ that will ensure that the weak solution is locally Hölder continuous. This question was considered in [LS] in the case of the Laplacian, and it was found that if $\mu[B(x, r)] \leq C r^{N-2+\varepsilon}$ for all balls $B(x, r)$, then the solution is locally Hölder continuous. We will prove that an analogous condition is necessary for equation (1.1) and also sufficient provided that $A$ satisfies a strong monotonicity condition. For this purpose, the following gradient estimate is crucial. This estimate was first used in [GZ] for investigating boundary regularity.

3.1. Theorem. Let $\mu$ be a nonnegative Radon measure on $\Omega$, and let $u \in$ $W_{\text {loc }}^{1, p}(\Omega), 1<p<N$, be a nonnegative, bounded solution of

$$
-\operatorname{div} A(x, u, \nabla u)+B(x, u, \nabla u)=\mu
$$


with structure (1.7), (1.8), (1.9). Then

$$
r^{p-N} \int_{B(x, r / 4)}|\nabla u|^{p} d x \leq C[\lambda(r / 2)-\lambda(r)+\rho(r)]^{p-1},
$$

where $\lambda(r)=\inf _{B(x, r)} u$ and $C$ depends on the structure and the bound for $u$. Proof. Since $\mu$ is a nonnegative measure, $u$ is therefore a weak supersolution of the equation

$$
-\operatorname{div} A(x, u, \nabla u)+B(x, u, \nabla u)=0 .
$$

First, consider an arbitrary weak supersolution $v$ of (3.2). It follows from the energy estimate in Lemma 2.3 and the techniques of [GZ] that

$$
\int v^{-\beta-1} \eta^{p}|\nabla v|^{p} d x \leq C\left(1+\beta^{-p}\right) \int v^{p-\beta-1}\left\{\eta^{p}+|\nabla \eta|^{p}\right\} d x
$$

whenever $\beta>0$ and $\eta$ is a cut-off function. Let $\theta$ be a positive number such that $1<(1-\theta) p<N /(N-p)$. Then,

$$
\begin{aligned}
\int_{B(x, r / 2)}|\nabla v|^{p-1} d x= & \int_{B(x, r / 2)}\left(v^{-(1-\theta)}|\nabla v|\right)^{p-1}\left(v^{(1-\theta)(p-1)}\right) d x \\
\leq & {\left[\int_{B(x, r / 2)}\left(v^{-(1-\theta) p}|\nabla v|^{p}\right) d x\right]^{(p-1) / p} } \\
& \times\left[\int_{B(x, r / 2)}\left(v^{(1-\theta)(p-1) p}\right) d x\right]^{1 / p} .
\end{aligned}
$$

It follows from (3.3) that the first factor is bounded by

$$
C r^{1-p}\left[\int_{B(x, r)} v^{\theta p} d x\right]^{(p-1) / p}
$$

provided $\eta$ is taken so that $\eta=1$ on $B(x, r / 2)$ with support contained in $B(x, r)$. Then, by Theorem 2.2 we have

$$
\begin{aligned}
& r^{p-N-1} \int_{B(x, r / 2)}|\nabla v|^{p-1} d x \\
& \quad \leq C(\lambda(r / 2)+\rho(r))^{((p-1) / p)(p-(1-\theta) p)}(\lambda(r / 2)+\rho(r))^{(1 / p)(1-\theta)(p-1) p} \\
& \leq C(\lambda(r / 2)+\rho(r))^{(p-1)} .
\end{aligned}
$$

From the analysis in Lemma 2.3, we have

$$
\begin{aligned}
\int_{B(x, r / 4)}|\nabla v|^{p} d x & \leq C\left\{r^{-1} \int_{B(x, r / 2)}|\nabla v|^{p-1}+r^{N-p} \rho(r)^{p-1}\right\} \\
& \leq C\left\{[\lambda(r / 2)+\rho(r)]^{p-1} r^{N-p}+r^{N-p} \rho(r)^{p-1}\right\} .
\end{aligned}
$$

Now, to establish the theorem, let $v=u-\inf _{B(x, r)} u$ and observe that $v$ is a supersolution of an equation with structure similar to that of (3.2).

This estimates leads immediately to the following necessary condition for a solution to be Hölder continuous. 
3.2. Theorem. If $u \in W_{\mathrm{loc}}^{1, p}(\Omega), 1<p<N$, is a nonnegative, bounded solution of

$$
-\operatorname{div} A(x, u, \nabla u)+B(x, u, \nabla u)=\mu
$$

that is locally Hölder continuous on $\Omega$, then there exists $\varepsilon>0$ such that

$$
\mu[B(x, r)] \leq C r^{N-p+\varepsilon}
$$

whenever $B(x, 16 r) \subset \Omega$. Here $C$ depends on the structure of the equation while $\varepsilon$ depends also on the Hölder exponent of $u$.

Proof. With $B(x, r)$ as in the statement of the theorem, let $\varphi$ be a smooth function that is 1 on $B(x, r)$ and with support contained in $B(x, 2 r)$. Then appealing to the structure (1.8), (1.9), and the previous theorem, we obtain

$$
\begin{aligned}
\mu[B(x, r)] & \leq \int \varphi d \mu \\
& \leq \frac{C}{r}\left[\int_{B(x, 2 r)}|A(x, u, \nabla u)| d x\right]+C \int_{B(x, 2 r)}|B(x, u, \nabla u)| d x \\
& \leq \frac{C}{r}\left[\int_{B(x, 2 r)}|\nabla u|^{p-1}+\left|a_{0}\right| d x\right]+C \int_{B(x, 2 r)}\left[|\nabla u|^{p}+\left|f_{0}\right|\right] d x \\
& \leq C\left[r^{N-p}[\lambda(8 r)-\lambda(16 r)+\rho(r)]^{p-1}\right. \\
& \left.\quad+r^{N-p}[\lambda(8 r)-\lambda(16 r)+\rho(r)]^{p-1}+r^{N-p} \rho(r)^{p-1}\right] .
\end{aligned}
$$

Now using the assumption that $u$ is locally Hölder continuous and that $\rho(r)$ is bounded by a positive power of $r$, the conclusion follows.

We now proceed to determine conditions under which the converse of the preceding theorem holds. We first prove that solutions of

$$
-\operatorname{div} A(x, u, \nabla u)+B(x, u, \nabla u)=\mu
$$

are locally bounded. For this, we require the following result of Adams [A].

3.3. Theorem. Let $\mu$ be a nonnegative Radon measure supported in $\Omega$ such that for all $x \in R^{n}$ and $0<r<\infty$, there is a constant $M$ with the property that

$$
\mu[B(x, r)] \leq M r^{a}
$$

where $a=q(N / p-1), 1<p<q<\infty$, and $p<N$. If $u \in W_{0}^{1, p}(\Omega)$, then

$$
\left(\int_{\Omega}|u|^{q} d \mu\right)^{1 / q} \leq C M^{1 / q}\|\nabla u\|_{p}
$$

where $C=C(p, q, N)$.

3.4. Theorem. If $u \in W_{\mathrm{loc}}^{1, p}(\Omega), 1<p<N$, is a weak solution of

$$
-\operatorname{div} A(x, u, \nabla u)+B(x, u, \nabla u)=\mu,
$$


with structure (1.7), (1.8), and (1.10) where $\mu$ is a nonnegative Radon measure supported in $\Omega$ with the property that for some $\varepsilon>0$,

$$
\mu[B(x, r)] \leq M r^{N-p+\varepsilon}
$$

for all $x \in \Omega$ and $0<r<\infty$. For $0<\sigma<1$ and $B\left(x, r^{*}\right) \subset \Omega$, there exists $\alpha, \beta>0$ such that

$$
\sup _{B\left(x, \sigma r^{*}\right)} u \leq \frac{C}{r^{* 1 / \alpha}}\left\{\int_{B\left(x, r^{*}\right)}|u|^{p} d x+\int_{B\left(x, r^{*}\right)}|u|^{p} d \mu\right\}^{1 / p}+C r^{* N \beta} .
$$

Proof. To simplify notation, let $B(x, r)$ be denoted simply by $B(r)$. For a value of $k$ that will determined later, we set

$$
k_{i}=k\left(1-2^{-i}\right), \quad i=0,1,2, \ldots,
$$

and

$$
\begin{gathered}
r_{i}=\sigma r^{*}+2^{-i} r^{*}(1-\sigma), \\
\bar{r}_{i}=\frac{1}{2}\left(r_{i}+r_{i+1}\right)=\sigma r^{*}+\frac{3}{4} 2^{-i} r^{*}(1-\sigma), \quad i=0,1,2, \ldots
\end{gathered}
$$

We consider the corresponding balls

$$
B_{i}=B\left(r_{i}\right), \quad \widetilde{B}_{i}=B\left(\bar{r}_{i}\right),
$$

and observe that

$$
\left(r_{i}-r_{i+1}\right)^{-1}=\frac{2^{i+1}}{r^{*}(1-\sigma)} .
$$

Finally, we denote by $\zeta_{i}$ the cut-off function whose support is contained in $\widetilde{B}_{i}$ such that $\zeta_{i}=1$ on $B_{i+1}$ and $\left|\nabla \zeta_{i}\right| \leq 2^{i+2} / r^{*}(1-\sigma)$.

Let

$$
A_{i}=B\left(r_{i}\right) \cap\left\{\left(u-k_{i+1}\right)^{+}>0\right\} .
$$

If the test function $\varphi=\zeta_{i}^{p}\left(u-k_{i+1}\right)^{+}$is employed, by an analysis similar to that used to establish Lemma 2.3 , we obtain the fundamental energy estimate:

$$
\begin{aligned}
\int_{B_{i+1}}\left|\nabla\left(u-k_{i+1}\right)^{+}\right|^{p} d x \leq C\left[\frac{1}{(1-\sigma)^{p}\left(r^{*}\right)^{p}} \int_{\widetilde{B}_{i}}\left|\left(u-k_{i+1}\right)^{+}\right|^{p} d x\right. \\
\left.\quad+\int_{\widetilde{B}_{i}}\left|\left(u-k_{i+1}\right)^{+}\right|^{p} d \mu+\left(k^{p}+\lambda\right)\left|A_{i}\right|+\mu\left(A_{i}\right)\right]
\end{aligned}
$$

because

$$
\begin{aligned}
\int \varphi d \mu & =\int_{\widetilde{B}_{i}} \zeta_{i}^{p}\left(u-k_{i+1}\right)^{+} d \mu \leq \int_{\widetilde{B}_{i}}\left(u-k_{i+1}\right)^{+} d \mu \\
& \leq\left(\int_{\widetilde{B}_{i}}\left|\left(u-k_{i+1}\right)^{+}\right|^{p} d \mu\right)^{1 / p} \mu\left(A_{i}\right)^{1-1 / p} \\
& \leq C\left[\int_{\widetilde{B}_{i}}\left|\left(u-k_{i+1}\right)^{+}\right|^{p} d \mu+\mu\left(A_{i}\right)\right] .
\end{aligned}
$$


If $q$ is defined by $N-p+\varepsilon=(q / p)(N-p)$, then the previous result of Adams implies that $\varphi \in L^{q}(d \mu)$. Note that $q / p>1$. Now

$$
\begin{aligned}
\int_{B_{i+1}}\left|\left(u-k_{i+1}\right)^{+}\right|^{p} d \mu \leq \int_{\widetilde{B}_{i}}\left|\left(u-k_{i+1}\right)^{+} \zeta_{i}\right|^{p} d \mu \\
\leq\left(\int_{\widetilde{B}_{i}}\left|\left(u-k_{i+1}\right)^{+} \zeta_{i}\right|^{q} d \mu\right)^{p / q} \mu\left(A_{i}\right)^{1-p / q} \\
\leq C\left\{\int_{\widetilde{B}_{i}}\left|\nabla\left(u-k_{i+1}\right)^{+}\right|^{p} d x+\int_{\widetilde{B}_{i}}\left|\left(u-k_{i+1}\right)^{+}\right|^{p}|\nabla \zeta|_{i}^{p} d x\right\} \mu\left(A_{i}\right)^{1-p / q} \\
\leq \frac{C 2^{i p}}{(1-\sigma)^{p} r^{* p}}\left\{\int_{B_{i}}\left|\left(u-k_{i}\right)^{+}\right|^{p} d x\right. \\
\left.\quad+\int_{B_{i}}\left|\left(u-k_{i}\right)^{+}\right|^{p} d \mu+\left(k^{p}+\lambda\right)\left|A_{i}\right|+\mu\left(A_{i}\right)\right\} \mu\left(A_{i}\right)^{1-(p / q)} .
\end{aligned}
$$

Now

$$
\mu\left(A_{i}\right) \leq 22^{p i} k^{-p} \int_{B_{i}}\left|\left(u-k_{i}\right)^{+}\right|^{p} d \mu \leq 22^{p i} Y_{i}
$$

and

$$
\left|A_{i}\right| \leq 22^{p i} k^{-p} \int_{B_{i}}\left|\left(u-k_{i}\right)^{+}\right|^{p} d x \leq 22^{p i} Y_{i},
$$

where $Y_{i}$ is defined by

$$
Y_{i}=k^{-p} \int_{B_{i}}\left|\left(u-k_{i}\right)^{+}\right|^{p} d x+k^{-p} \int_{B_{i}}\left|\left(u-k_{i}\right)^{+}\right|^{p} d \mu .
$$

Hence, if $\beta$ is chosen so that $0<\beta<1-(p / q)$, it follows from (3.7) that

$$
\begin{aligned}
& k^{-p} \int_{B_{i+1}}\left|\left(u-k_{i+1}\right)^{+}\right|^{p} d \mu \\
& \leq \frac{C 2^{i p}}{(1-\sigma)^{p} r^{* p}}\left[\left(Y_{i}\right)\left(2^{p i} Y_{i}\right)^{1-p / q}+\left(\frac{\left(k^{p}+\lambda\right) r^{N \beta}}{k^{p}}\right)\left(2^{p i} Y_{i}\right)^{1-\beta}\left(2^{p i} Y_{i}\right)^{1-p / q}\right. \\
& \left.+\left(2^{p i} Y_{i}\right)\left(2^{p i} Y_{i}\right)^{1-(p / q)}\right] .
\end{aligned}
$$

If $k$ is chosen so that $k^{p} \geq \lambda r^{* N \beta}$ and $k \geq\|u\|_{p ; B_{0}}$, it therefore follows that

$$
k^{-p} \int_{B_{i+1}}\left|\left(u-k_{i+1}\right)^{+}\right|^{p} d \mu \leq C \frac{C 2^{i p}}{(1-\sigma)^{p} r^{* p}} Y_{i}^{1+\alpha}
$$

for some $\alpha>0$. Now, repeating the previous analysis with the term

$$
\int_{B_{i+1}}\left|\left(u-k_{i+1}\right)^{+}\right|^{p} d \mu
$$


replaced by

$$
\int_{B_{i+1}}\left|\left(u-k_{i+1}\right)^{+}\right|^{p} d x
$$

under the assumption that $q<N p /(N-p)$, we obtain the following inequality:

$$
\begin{aligned}
\int_{B_{i+1}}\left|\left(u-k_{i+1}\right)^{+}\right|^{p} d x & \\
\leq \frac{C 2^{i p}}{(1-\sigma)^{p} r^{* p}}\left\{\int_{B_{i}}\left|\left(u-k_{i}\right)^{+}\right|^{p} d x\right. & +\left.\int_{B_{i}}\left(u-k_{i}\right)^{+}\right|^{p} d \mu \\
& \left.+\left(k^{p}+\lambda\right)\left|A_{i}\right|+\mu\left(A_{i}\right)\right\}\left|A_{i}\right|^{1-p / q} .
\end{aligned}
$$

Hence

$$
k^{-p} \int_{B_{i+1}}\left|\left(u-k_{i+1}\right)^{+}\right|^{p} d x \leq \frac{C}{(1-\sigma)^{p}} \frac{2^{p i}}{r^{* p}} Y_{i}^{1+\alpha} .
$$

From (3.8) and (3.9), we have

$$
Y_{i+1} \leq \frac{C b^{i}}{(1-\sigma)^{p} r^{* p}} Y_{i}^{1+\alpha}
$$

where $b=2^{p}>1$ and $k^{p} \geq \lambda r^{* N \beta}$ and $k \geq\|u\|_{p ; B_{0}}$. The recursion lemma of [LU, p. 66] implies that $Y_{i} \rightarrow 0$ provided

$$
Y_{0} \leq C(p)\left((1-\sigma) r^{*}\right)^{p / \alpha}
$$

or

$$
Y_{0}=k^{-p} \int_{B\left(r^{*}\right)}|u|^{p} d x+k^{-p} \int_{B\left(r^{*}\right)}|u|^{p} d \mu \leq C(p, i)\left((1-\sigma) r^{*}\right)^{p / \alpha}
$$

which suffices to reach the desired conclusion.

3.5. Remark. By applying the interpolation technique of [DT], it is easy to see that the above result remains valid with $p$ replaced by $q$ for any $q>0$.

We now are able to establish regularity with operators $A$ of the following type.

3.6. Definition. We say that the operator $A(x, \eta, \xi)$ is strongly monotonic in $\Omega$ provided

$$
\left(A(x, \eta, \xi)-A\left(x, \eta, \xi^{\prime}\right)\right) \cdot\left(\xi-\xi^{\prime}\right) \geq C\left|\xi-\xi^{\prime}\right|^{p}
$$

for all $x \in \Omega, \eta \in \mathbf{R}$, and all $\xi, \xi^{\prime} \in \mathbf{R}^{N}$.

3.7. Theorem. Let $u \in W_{\mathrm{loc}}^{1, p}(\Omega), 1<p<N$, be a weak solution of

$$
-\operatorname{div} A(x, u, \nabla u)+B(x, u, \nabla u)=\mu,
$$

where $\mu$ is a nonnegative measure with the property that $\mu[B(x, r)] \leq C r^{N-p+\varepsilon}$ for all balls $B(x, r)$ with $B(x, 2 r) \subset \Omega$. If we assume structure (1.7), (1.8), 
and (1.10), and that $A$ is strongly monotonic in $\Omega$, then $u$ is locally Hölder continuous on $\Omega$.

Proof. Let $\Omega_{1}$ be an bounded, open subset whose closure is contained in $\Omega$. Let $x_{0} \in \Omega_{1}$ and consider $0<r<\operatorname{dist}\left(x_{0}, \partial \Omega_{1}\right)$. Since by Theorem 3.4 the solution $u$ is bounded in $\Omega_{1}$, we may consider $A(x, u(x), \xi)$ as $A(x, \xi)$ and normalize the structure by taking $\min \nu_{0}=1$, thus obtaining

$$
|A(x, \xi)| \leq C_{0}|\xi|^{p-1}+a_{0}, \quad A(x, \xi) \cdot \xi \geq|\xi|^{p}-\nu_{1},
$$

for all $(x, \xi) \in B\left(x_{0}, r\right) \times \mathbf{R}^{N}$, where $B\left(x_{0}, r\right) \subset \Omega_{1}, a_{0} \in L^{q_{1}}\left[B\left(x_{0}, r\right)\right]$, and $\nu_{1} \in L^{q_{2}}\left[B\left(x_{0}, r\right)\right]$ with $q_{1}>N /(p-1), q_{2}>n / p$. Now let $v \in W^{1, p}\left[B\left(x_{0}, r\right)\right]$ be defined as a solution of

$$
\operatorname{div} A(x, \nabla v)=0,
$$

where $u-v \in W_{0}^{1, p}\left[B\left(x_{0}, r\right)\right]$. The existence of $v$ is provided by [L, pp. 182183] and because $u$ is bounded on the closure of $B\left(x_{0}, r\right)$, we may appeal to [SE, Theorem 3] to conclude that $v$ is bounded on $B\left(x_{0}, r\right)$. Using the strong monotonicity of $A$ and the fact that $u-v$ is a test function, we obtain

$$
\text { (3.10) } \begin{aligned}
\int_{B\left(x_{0}, r\right)} & |\nabla(u-v)|^{p} \leq C \int_{B\left(x_{0}, r\right)}(A(x, u, \nabla u)-A(x, u, \nabla v)) \cdot \nabla(u-v) \\
= & C\left\{\int_{B\left(x_{0}, r\right)} B(x, u, \nabla u)(u-v) d x+\int_{B\left(x_{0}, r\right)}(u-v) d \mu\right\} \\
\leq & C\left\{\int_{B\left(x_{0}, r\right)}|\nabla u|^{p-1}|u-v| d x+\int_{B\left(x_{0}, r\right)} f_{0}(u-v) d x+r^{N-p+\varepsilon}\right\} .
\end{aligned}
$$

With the help of Young's and Poincaré's inequality, there exists $C$ depending on $p$ and the structure such that for any real number $\gamma>0$,

$$
\begin{gathered}
C \int_{B\left(x_{0}, r\right)}|\nabla u|^{p-1}|u-v| d x \leq\|\nabla u\|_{p ; B\left(x_{0}, r\right)}^{p-1}\|u-v\|_{p ; B\left(x_{0}, r\right)} \\
\quad \leq\|\nabla u\|_{p ; B\left(x_{0}, r\right)}^{p-1} \cdot r\|\nabla(u-v)\|_{p ; B\left(x_{0}, r\right)} \\
\quad \leq C(\gamma) r^{p^{\prime}} \int_{B\left(x_{0}, r\right)}|\nabla u|^{p}+\gamma \int_{B\left(x_{0}, r\right)}|\nabla(u-v)|^{p} d x .
\end{gathered}
$$

Consequently, (3.10) can be written as

$$
\begin{aligned}
\int_{B\left(x_{0}, r\right)} & |\nabla(u-v)|^{p} \\
\leq C & \left\{r^{p^{\prime}} \int_{B\left(x_{0}, r\right)}|\nabla u|^{p} d x+\int_{B\left(x_{0}, r\right)} f_{0}(u-v) d x+r^{N-p+\varepsilon}\right\} .
\end{aligned}
$$

As for the second term in (3.12), the local boundedness of $(u-v)$ implies

$$
\int_{B\left(x_{0}, r\right)} f_{0}(u-v) d x \leq C r^{N-p+\varepsilon^{\prime}}
$$


where $\varepsilon^{\prime}$ depends on $q>N / p$. Thus, we conclude from (3.12) that there are constants $C$ and $C^{\prime}$ depending only on the given data such that

$$
\int_{B\left(x_{0}, r\right)}|\nabla(u-v)|^{p} d x \leq C r^{p^{\prime}} \int_{B\left(x_{0}, r\right)}|\nabla u|^{p} d x+C^{\prime} r^{N-p+\varepsilon}
$$

for some $\varepsilon>0$. Since $v$ is locally Hölder continuous on compact subsets of $B\left(x_{0}, r\right)$, it follows from the fundamental energy estimate, Lemma 2.3 , and an elementary scaling argument, that

$$
\int_{b\left(x_{0}, \rho\right)}|\nabla v|^{p} d x \leq C\left(\frac{\rho}{r}\right)^{N-p+\varepsilon^{\prime}} \int_{B\left(x_{0}, r\right)}|\nabla v|^{p} d x
$$

for all $0<\rho<r$ and some $\varepsilon^{\prime}>0$.

We now proceed to obtain a bound for

$$
\int_{B\left(x_{0}, r\right)}|\nabla v|^{p} d x
$$

Since

$$
0=\int A(x, \nabla v) \cdot \nabla(u-v) d x=\int A(x, u, \nabla v) \cdot \nabla(u-v) d x,
$$

it follows that there exist $C$ and $C^{\prime}$ depending on (1.7), (1.8), and (1.10) such that

$$
\begin{aligned}
& \int_{B\left(x_{0}, r\right)}|\nabla v|^{p} d x \leq \int_{B\left(x_{0}, r\right)}\left(A(x, u, \nabla v) \cdot \nabla u+\nu_{1}\right) d x \\
& \leq C \int_{B\left(x_{0}, r\right)}|\nabla v|^{p-1}|\nabla u| d x+C \int_{B\left(x_{0}, r\right)}\left(a_{0}|\nabla u|+\nu_{1}\right) d x \\
& \leq \frac{1}{2} \int_{B\left(x_{0}, r\right)}|\nabla v|^{p} d x+C^{\prime} \int_{B\left(x_{0}, r\right)}|\nabla v|^{p} d x \\
&+C^{\prime} \int_{B\left(x_{0}, r\right)}|\nabla u|^{p} d x+C^{\prime}\left(\int_{B\left(x_{0}, r\right)}\left|a_{0}\right|^{q_{1}} d x\right)^{1 / s} \cdot r^{N-p+\varepsilon}+C^{\prime} r^{N-p+\varepsilon}
\end{aligned}
$$

since $q_{1}>N /(p-1)$ and $q_{2}>N / p$. Here $s=q_{1} / p^{\prime}$. Thus, there exists $C$ and $\varepsilon>0$ such that

$$
\int_{B\left(x_{0}, r\right)}|\nabla v|^{p} d x \leq C\left\{\int_{B\left(x_{0}, r\right)}|\nabla u|^{p} d x+r^{N-p+\varepsilon}\right\} .
$$

Now $u=v+(u-v)$ and thus, from (3.14), (3.15), (3.13), we have

$$
\begin{aligned}
C \int_{B\left(x_{0}, \rho\right)}|\nabla u|^{p} d x & \leq \int_{B\left(x_{0}, \rho\right)}|\nabla v|^{p} d x+\int_{B\left(x_{0}, \rho\right)}|\nabla(u-v)|^{p} d x \\
& \leq\left(\frac{\rho}{r}\right)^{N-p+\varepsilon} \int_{B\left(x_{0}, r\right)}|\nabla v|^{p} d x+\int_{B\left(x_{0}, r\right)}|\nabla(u-v)|^{p} d x \\
& \leq\left[\left(\frac{\rho}{r}\right)^{N-p+\varepsilon}+r^{p^{\prime}}\right] \int_{B\left(x_{0}, r\right)}|\nabla v|^{p} d x+r^{N-p+\varepsilon}
\end{aligned}
$$


for every $0<\rho<r$. We now are able to invoke the technique in [G, p. 170] to conclude that there exists $\varepsilon_{0}>0$ such that if

$$
r^{p-N} \int_{B\left(x_{0}, r\right)}|\nabla u|^{p} d x \leq \varepsilon_{0}
$$

then there exists $\varepsilon>0$ and a constant $C$ depending only on the structure such that

$$
\rho^{p-N} \int_{B\left(x_{0}, \rho\right)}|\nabla u|^{p} d x \leq C\left(\frac{\rho}{r}\right)^{\varepsilon}
$$

for all $0<\rho<r$. As in [G] we conclude that $u$ is locally Hölder continuous in $B\left(x_{0}, r\right)$. This conclusion is based on (3.16) which is know to hold at all points $x_{0} \in \Omega_{1}$ by Theorem 3.1.

Another result for equations of divergence form was first proved in [ME] which states that a solution $u \in W^{1, p}(\Omega)$ has the property that $u \in W^{1, q}(\Omega)$ for some $q>p$. A similar result is valid for the type of equations we are considering.

3.8. Theorem. Let $u \in W_{\mathrm{loc}}^{1, p}(\Omega)$ be a weak solution of (1.1) with structure (1.7), (1.8), (1.10) and with $T \in W_{\mathrm{loc}}^{-1, p^{\prime}+\varepsilon}(\Omega)$ for some $\varepsilon>0$. Then there exists $q>p$ such that $u \in W_{\text {loc }}^{1, q}(\Omega)$. Moreover, for $B(x, r) \subset \Omega$,

$$
\left(f_{B(x, r / 2)}|\nabla u|^{q} d x\right)^{1 / q} \leq C\left(\left(f_{B(x, r)}|\nabla u|^{p} d x\right)^{1 / p}+\left(f_{B(x, r)} h^{q} d x\right)^{1 / q}\right),
$$

where $h=\left(a_{0}^{p^{\prime}}+|g|^{p^{\prime}}\right)^{1 / p}, \operatorname{div} g=T+f_{0}$.

Proof. For fixed $x$ such that $B(x, r) \subset \Omega$, let

$$
\bar{u}(r)=f_{B(x, r)} u(y) d y
$$

and define a test function by $\varphi=\eta^{p}(u-\bar{u}(r))$, where $\eta$ is a smooth cutoff function such that $\eta \equiv 1$ on $B(x, r / 2)$ and $\operatorname{spt} \eta \subset B(x, r)$. With $\varphi$ employed as a test function in (1.1), we obtain

$$
\begin{aligned}
& \int \eta^{p}|\nabla u|^{p} d x \\
& \leq C\left\{\int|\nabla u|^{p-1}|\nabla u| \eta^{p-1}(u-\bar{u}(r)) d x\right. \\
& \left.\quad+\int a_{0}|\nabla \eta| \eta^{p-1}(u-\bar{u}(r)) d x+\int c_{1}|\nabla u|^{p-1} \eta^{p}(u-\bar{u}(r)) d x\right\} \\
& \quad+\left\langle\operatorname{div} g, \eta^{p}(u-\bar{u}(r))\right\rangle,
\end{aligned}
$$

where $g \in L^{p^{\prime}+\varepsilon}\left(\Omega ; \mathbf{R}^{N}\right)$, $\operatorname{div} g=T+f_{0}$, and $C$ depends only on the structure. The last term can be replaced by

$$
C \int|g|\left|\nabla\left[\eta^{p}(u-\bar{u}(r))\right]\right| d x .
$$


Now the analysis proceeds as in [EM] to establish the conclusion or see [G, Theorem 2.2, p. 138] for an easy exposition.

This result is especially relevant for our purposes because of the following theorem of Hedberg and Wolff [HW].

3.9. Theorem. Let $p>1$ and $k p<N$. If $\mu$ is a Radon measure, then $\mu \in\left(W^{k, p}\left(\mathbf{R}^{N}\right)\right)$ if and only if

$$
\int_{\mathbf{R}^{N}} \int_{0}^{1}\left(\frac{\mu[B(y, r)]}{r^{N-k p}}\right)^{1 /(p-1)} \frac{d r}{r} d \mu(y)<\infty .
$$

As a consequence of Theorems 3.8 and 3.9 we have

3.10. Corollary. Let $u \in W_{\mathrm{loc}}^{1, p}(\Omega)$ be a weak solution of

$$
-\operatorname{div} A(x, u, \nabla u)+B(x, u, \nabla u)=\mu
$$

where $\mu$ is a nonnegative measure supported in $\Omega$ with the property that $\mu[B(x, r)] \leq C r^{N-p+\varepsilon}$ for all balls $B(x, r) \subset \Omega$. If we assume structure (1.7), $(1.8)$, and (1.10), then there exists $q>p$ such that $u \in W_{\text {loc }}^{1, q}(\Omega)$. Moreover, for $B(x, r) \subset \Omega$,

$$
\left(f_{B(x, r / 2)}|\nabla u|^{q} d x\right)^{1 / q} \leq C\left(\left(f_{B(x, r)}|\nabla u|^{p} d x\right)^{1 / p}+\left(f_{B(x, r)} h^{q} d x\right)^{1 / q}\right),
$$

where $h$ is as in Theorem 3.8.

As another corollary of the above results, consider the variational inequality (1.5) which is assumed to hold for all $\varphi \in W^{1, p}(\Omega)$ for which $\varphi \geq \psi-u$ a.e., where $\psi \in L^{\infty}(\Omega)$ is the obstacle. Then there is a measure $\mu$ associated with this problem as explained in (1.6). If in addition it is assumed that $\psi \in$ $C_{\text {loc }}^{0, \alpha}(\Omega)$, then referring to either [MZ] or [RT] we have that $u \in C_{\text {loc }}^{0, \alpha^{\prime}}(\Omega)$. It follows from Theorem 3.2 that for all $B(x, r) \subset \Omega, \mu\left[(B(x, r)] \leq C r^{N-p+\varepsilon}\right.$ for some $\varepsilon>0$. By appealing to the previous corollary, we are led to the following result.

3.11. Corollary. If $u \in W_{\mathrm{loc}}^{1, p}(\Omega)$ satisfies the variational inequality

$$
\int A(x, u, \nabla u) \cdot \nabla \varphi+B(x, u, \nabla u) \varphi d x \geq 0
$$

for all $\varphi \in W_{0}^{1, p}(\Omega)$ with the property that $\varphi \geq \psi-u$ a.e., where $\psi \in C_{\mathrm{loc}}^{0, \alpha}(\Omega)$, then there exists $q>p$ such that $u \in W_{\mathrm{loc}}^{1, q}(\Omega)$. Moreover, for $B(x, r) \subset \Omega$,

$$
\left(f_{B(x, r / 2)}|\nabla u|^{q} d x\right)^{1 / q} \leq C\left(\left(f_{B(x, r)}|\nabla u|^{p} d x\right)^{1 / p}+\left(f_{B(x, r)} h^{q} d x\right)^{1 / q}\right),
$$

where $h$ is as in Theorem 3.8 .

We conclude with a result that extends Theorem 3.4 and resembles the classical Harnack inequality, (2.10). 
3.12. Theorem. Let $u \in W_{\mathrm{loc}}^{1, p}(\Omega)$ be a nonnegative weak solution of

$$
-\operatorname{div} A(x, u, \nabla u)+B(x, u, \nabla u)=\mu
$$

with structure (1.7), (1.8), and (1.10), where $\mu$ is a nonnegative measure supported in $\Omega$ with the property that $\mu[B(x, r)] \leq C r^{N-p+\varepsilon}$ for all balls $B(x, r) \subset \Omega$. Then there exist $\alpha, \beta>0$ and $C$ depending on the structure and $\varepsilon$ such that for $0<r<1$,

$$
\sup _{B(x, r / 2)} u \leq C r^{-\alpha}\left\{\left[\inf _{B(x, r / 2)} u+\rho(r)\right]^{p-1}\right\}+C r^{\beta}, \quad \text { if } 1<p<2,
$$

and

$$
\sup _{B(x, r / 2)} u \leq C r^{-\alpha}\left\{\left[\inf _{B(x, r / 2)} u+\rho(r)\right]\right\}+C r^{\beta}, \quad \text { if } p \geq 2 .
$$

Proof. Choose $x \in \Omega$ and $0<r<1$ such that $B(x, 2 r) \subset \Omega$. With $\eta$ as a smooth cut-off function such that $\eta \equiv 1$ on $B(x, r)$ and spt $\eta \subset B(x, 2 r)$, we use the test function $\varphi=\eta^{p} u$ to obtain

$$
\begin{aligned}
\int_{B(x, r)} u d \mu \leq & \int \varphi d \mu=\int A(x, u, \nabla u) \cdot \nabla \varphi+B(x, u, \nabla u) \varphi d x \\
\leq & C\left\{\int|\nabla u|^{p-1} \eta^{p-1}|\nabla \eta| u d x+\int|\nabla u|^{p} \eta^{p} u d x\right. \\
& \left.\quad+\int \eta^{p} u a_{0} d x+\int \eta^{p} u|\nabla u|^{p-1} d x+\int \eta^{p} u f_{0} d x\right\} \\
\leq & C\left\{\frac{1}{r} \int_{B(x, 2 r)}|\nabla u|^{p-1}+\int_{B(x, 2 r)}|\nabla u|^{p} d x+r^{N-p} \rho(r)^{p-1}\right\} .
\end{aligned}
$$

Here we have used the fat that $u$ is locally bounded (Theorem 3.4) and $0<$ $r<1$. Appealing to Theorem 3.1, it follows that

$$
\int_{B(x, r)} u d \mu \leq \int \varphi d \mu \leq C\left\{[\lambda(4 r)-\lambda(8 r)+\rho(r)]^{p-1} r^{N-p}+r^{N-p} \rho(r)^{p-1}\right\} .
$$

In order to obtain a similar bound for $\int u \eta d x$, we use Theorem 2.2 and the fact that $u$ is locally bounded to conclude that

$$
\int_{B(x, r)} u d x \leq C \int_{B(x, r)} u^{p-1} d x \leq C r^{N}[\lambda(r / 2)+\rho(r)]^{p-1}
$$

when $1<p<2($ since $0<p-1<N(p-1) /(N-p)$ ) and that

$$
\int_{B(x, r)} u d x \leq C r^{N}[\lambda(r / 2)+\rho(r)]
$$

when $p \geq 2$ (since $N(p-1) /(N-p)>1)$. These conclusions along with (3.17), Theorem 3.4 and Remark 3.5 establish the result. 


\section{REFERENCES}

[A] D. Adams, Traces of potentials arising from translation invariant operators, Ann. Scuola Norm. Sup. Pisa 25 (1971), 203-217.

[DT] E. DiBenedetto and N. Trudinger, Harnack inequalities for quasi-minima of variational integrals, Ann. Inst. H. Poincaré. Anal. Non Linéaire 1 (1984), 295-308.

[G] M. Giaquinta, Multiple integrals in the calculus of variation and nonlinear elliptic systems, Ann. of Math. Stud., no. 105, Princeton Univ. Press, Princeton, N.J., 1983.

[GZ] R. Gariepy and W. P. Ziemer, A regularity condition at the boundary for solutions of quasilinear elliptic equations, Arch. Rational Mech. Anal. 67 (1977), 25-39.

[GT] D. Gilbarg and N. Trudinger, Elliptic partial differential equations of second order, 2nd ed., Springer, New York, 1983.

[HW] L. Hedberg and T. Wolff, Thin sets in nonlinear potential theory, Ann. Inst. Fourier (Grenoble) 23 (1983), 161-187.

[JM] F. John and L. Nirenberg, On functions of bounded means oscillation, Comm. Pure Appl. Math. 14 (1961), 415-426.

[LU] O. A. Ladyzhenskaya and N. N. Ural ' tseva, Linar and quasilinear elliptic equations, Academic Press, 1968.

[LS] H. Levy and G. Stampacchia, On the smoothness of superharmonics which solve the minimum problem, J. Analyse Math. 23 (1970), 227-236.

[L] J. L. Lions, Quelques méthodes de résolution des problèmes aux limites non lineaires, Dunod, Paris, 1969.

[ME] N. Meyers and A. Elcrat, Some results on regularity for solutions of nonlinear elliptic systems and quasi-regular funtions, Duke Math. J. 42 (1975), 121-136.

[MZ] J. H. Michael and W. P. Ziemer, Interior regularity for solutions of obstacle problems, Nonlinear Analysis, TMA, 10 (1986), 1427-1448.

[MRS1] B. Michaux, J. M. Rakotoson, and J. Shen, On the existence and regularity of solutions of a quasilinear mixed equations of Leray-Lions type, Preprint, Institute for Appl. Math. and Sci. Comp., \#8807, 1988; Acta Appl. Math. 12 (1988), 287-316.

[MRS2] __ On the approximation of a quasilinear mixed problem, Preprint, Institute for Appl. Math. and Sci. Comp., \#8808, 1988, MMAN (to appear).

[RA] J. M. Rakotoson, Rearrangement relatif dans le equations elliptiques quasilineaire avec un second membre distribution, J. Differential Equations 66 (1987), 391-419.

[RT] J. M. Rakotoson and R. Temam, Relative rearrangement in quasilinear variational elliptic inequalities, Indiana Univ. Math. J. 36 (1987).

[S] J. Serrin, Local behavior of solutions of quasilinear equations, Acta Math. 111 (1964), 247302.

[T] N. Trudinger, On Harnack type inequalities and their application to quasilinear elliptic equations, Comm. Pure Appl. Math. 20 (1967), 721-747.

Department of Mathematics, Indiana University, Bloomington, Indiana 47405 